\title{
A New Combined Filter-Wrapper Framework for Gene Subset Selection with Specialized Genetic Operators
}

\author{
Edmundo Bonilla Huerta, J. Crispín Hernández Hernández, \\ and L. Alberto Hernández Montiel \\ LITI, Instituto Tecnológico de Apizaco, \\ Av. Instituto Tecnológico s/n, 90300 Apizaco, Mexico \\ \{edbonn, josechh, luishm\}@itapizaco.edu.mx
}

\begin{abstract}
This paper introduces a new combined filter-wrapper gene subset selection approach where a Genetic Algorithm (GA) is combined with Linear Discriminant Analysis (LDA). This LDA-based GA algorithm has the major characteristic that the GA uses not only a LDA classifier in its fitness function, but also LDA's discriminant coefficients in its dedicated crossover and mutation operators. This paper studies the effect of these informed operators on the evolutionary process. The proposed algorithm is assessed on a several well-known datasets from the literature and compared with recent state of art algorithms. The results obtained show that our filter-wrapper approach obtains globally high classification accuracies with very small number of genes to those obtained by other methods.
\end{abstract}

Keywords: Microarray gene expression, Feature selection, Genetic algorithms, Linear Discriminant Analysis, Filter, Wrapper.

\section{Introduction}

The DNA Microarray is a tool that permits to monitor and to measure gene expression levels for tens of thousands of genes simultaneously under hundreds of biological conditions. This technology enables to consider cancer diagnosis based on gene expressions 12236 . This microarray technology enables clinicians and biologists to obtain the gene expression profile of tissue samples rapidly.

Microarray data is characterized with thousands of genes but with only a small number of samples available for analysis. Microarray data often contains many irrelevant and redundant features, which affect the speed and accuracy of most learning algorithms. The task of selecting the "best" feature subset is known as feature selection, sometimes as variable selection or subset selection [4].

Feature selection consists to select a minimal subset of $m$ features from the original set of $n$ features $(m \ll n)$. Normally, a feature selection method consists of four components: a search mechanism, an evaluation function, a stopping criterion, and a validation procedure. 
In this paper, we propose a new combined filter-wrapper approach for gene subset selection for microarray data classification in two stages. In the first stage is proposed a filter for individual gene ranking. In the second stage a wrapper is proposed where Fisher's Linear Discriminant Analysis (LDA) is used to provide useful information to a Genetic Algorithm (GA) for an efficient exploration of gene subsets space. LDA has been used for several classification problems and recently for microarray data 7,10,11.

This paper is organized as follows: Section 2 describes the state of the art of feature selection. In Section 3 The General Genetic algorithm procedure is discussed in detail. Experimental results are shown in Section 4 and conclusions are drawn in Section 5.

\section{State of the Art}

Feature selection methods are separated into three main families [12]: 1) the filter approach, 2) the wrapper approach and 3) the embedded approach.

The filter approach uses statistical information to filter out irrelevant features. All features are first ranked and then a classifier is build by selecting the highest ranking features. In most cases, the selection relies on an individual evaluation of each feature 613, therefore this method ignores interactions among genes.

The wrapper approach relies on a classification algorithm that is used as a black box to explore the space of features subset to evaluate each candidate subset; the quality of a candidate subset is measured by the performance of the classifier obtained on the training data, using, for example a cross validation process. Finally, the feature subset that achieves highest performance is chosen. Wrapper methods are generally computation intensive since the classifier must be trained for each candidate subset. For this reason, several strategies can be considered to explore the space of possible subsets. In the context of microarray data, several wrapper methods have been proposed using uncorrelated discriminant analysis [10, generalized discriminant analysis [31, null space 11], diagonal covariance matrices [24] and linear discriminant analysis using pseudo-inverse [5].

Finally, in embedded methods, a inductive algorithm is used as feature selector and classifier. A representative work of this approach is the method that uses support vector machines with recursive feature elimination (SVM/RFE) [18]. The selection is based on a ranking of the genes and, at each step, the gene with the smallest ranking criterion is eliminated. The ranking criterion is obtained from the weights of a SVM trained on the current set of genes. In this sense, embedded methods are similar to wrapper methods. There are other variants of these approaches, see [1920 for two examples. In [21, the authors propose a forward search approach based in two steps. In the first a filter method is used as a pre-selection step, all features are ranked. In the second step a wrapper method is used for feature selection by building $N$ attributes subsets. These subsets are evaluated using the so-called Rank-Search algorithm, which is very fast, but gives very large feature subsets. 


\section{General GA Procedure}

Our approach first extracts a set of interesting genes (about $p=150$ genes) by a filter method in order to limit the search space. Then we use a dedicated Genetic Algorithm (GA) to determine a small subset of genes that allows high classification accuracy. Contrary to most existing GAs for gene selection that rely essentially on random genetic operators, we devised a problem specific GA that takes into account useful knowledge of the gene selection and classification problem. Indeed, our GA uses a LDA classifier to assess the fitness of a given candidate gene subset and LDA's discriminate coefficients in its crossover and mutation operators.

This paper first studies the impact of these informed operators on the evolutionary process. We present experimental evidence about the performance of our dedicated crossover operator when compared with two conventional crossover operators.

To evaluate the usefulness of the proposed approach, we carry out extensive experiments on seven public datasets and compare our results with 13 best performing algorithms from the literature. We observe that our approach is able to achieve a high prediction accuracy (from $81 \%$ to 100\%) with a very small number of informative genes (from 2 to 19).

\subsection{Filter Method}

Different feature selection approaches have been applied to gene selection for cancer classification (Fisher ratio, Wilcoxon test, Information Gain, Relief-F, T-statistics, entropy-based, BW ratio, etc.).

The $\mathbf{B W}$ ratio, introduced by Dudoit et al. [7, is the ratio of between-group to within-group sums of squares. For a gene $j$, the ratio is formally defined by:

$$
B W(j)=\frac{\sum_{i} \sum_{k} I\left(y_{i}=k\right)\left(\bar{x}_{k j}-\bar{x}_{j}\right)^{2}}{\sum_{i} \sum_{k} I\left(y_{i}=k\right)\left(x_{i j}-\bar{x}_{k j}\right)^{2}}
$$

where $I($.$) denotes the indicator function, equaling 1$ if the condition in parentheses is true, and 0 otherwise. $\bar{x}_{j}$ and $\bar{x}_{k j}$ denote respectively the average expression level of the gene $j$ across all samples and across samples belonging to class $k$ only and finally $y_{i}$ denotes a class label. This filter method considers the most discriminatory genes those that have the highest scores. We use this ranking-based method to identify differentially expressed genes in two tissue types.

In our filter-wrapper framework, we use three filters : between-group to withingroup sums of squares (BW), t-statistic (TT), and Wilcoxon test (WT) for a comparative analysis.

\subsection{Wrapper Method}

We describe now the wrapper method based in a genetic algorithm and a classifier LDA (GALDA) of the general model for gene selection and classification. The 
GA is designed for discovering good gene subsets by using genetic operators specialized. The LDA-based classifier is used to ensure the fitness evaluation of each candidate gene subset. The basic components of our GA are detailed in this section.

Encoding representation and initialization. In our GA, a chromosome encodes more information than a classic GA, because the chromosomes are formed by two parts of same length: 1) a binary vector and 2) a real-valued vector (as shown in Figure 1). The first part represents a candidate gene subset (150), where each allele (bit) of the chromosome represents a gene. If an allele is " 1 " it means that this gene is kept in the gene subset and " 0 " indicates that the gene is not included. Each chromosome represents thus a gene subset. The chromosome length is equal to the number of $p$ genes pre-selected by the filter t-statistics (3.1). The second part of the chromosome is a real-valued vector that corresponds to the discriminant coefficients obtained by a LDA classifier for a gene $g_{i}$ which are useful for designing powerful crossover and mutation operators (described below).

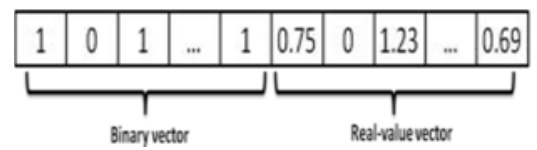

Fig. 1. Representation of a chromosome in our GA

The individual of the initial population are generated randomly according to a uniform distribution in such a way that each chromosome contains a number of genes ranging from $p=0.60$ to $p=0.75$. The population size is fixed at 100 in this work. The chromosomes of the current population $P$ are sorted according to the fitness function (see subsection 3.2). The best $10 \%$ chromosomes of $P$ are directly copied to the next population $P^{\prime}$ and removed from $P$. The remaining $90 \%$ chromosomes of $P^{\prime}$ are then generated by using specialized genetic operators.

Double fitness function. The fitness of a chromosome, i.e. a subset of genes, is assessed by two fitness functions: 1) the classification rate $\left(f_{1}\right)$, and 2 ) the number of selected genes in the each chromosome $\left(f_{2}\right)$. In the first part of the double fitness function, a subset of genes leading to a high classification rate is considered to be better than a subset leading to a low classification rate. In our case, the LDA classifier ensures this classification task. We apply a 10 fold cross validation to calculate the performance of the classifier LDA for a candidate gene subset. The second part of the fitness $\left(f_{2}\right)$ is calculated by the formula:

$$
f_{2}=\left(1-\frac{m_{\omega}}{p}\right)
$$


Where $m_{\omega}$ is the number of bits having the value " 1 " in the candidate gene subset $\omega, p$ is the length of the chromosome corresponding to the number of the pre-selected genes from the filter ranking. Then the fitness function $f$ is defined as the weighted aggregation of $f_{1}$ and $f_{2}$ as follows:

$$
f(I)=\alpha f_{1}(I)+(1-\alpha) f_{2} \text { subject to } 0<\alpha<1
$$

Where $\alpha$ is a parameter that allows us to allocate a relative importance factor to $f_{1}$ or $f_{2}$. Assigning to $\alpha$ a value greater than 0.5 will push the genetic search toward solutions of high classification accuracy (probably at the expense of having more selected genes). In contrast, using a small values of $\alpha$ helps the search toward small sized gene subsets. So varying $\alpha$ will change the search direction of the genetic algorithm.

Specialized genetic operator of crossover. We use the discriminant coefficients from a LDA classifier to design our specialized genetic operator of crossover. The crossover combines two parent chromosomes $I 1$ and $I 2$ to generate a new chromosome $I_{\text {new }}$ in such a way that 1 ) top ranking genes in both parents are preserved in the child and 2) the number of selected genes in the child $I_{n e w}$ is not greater than the number of selected genes in the parents. The first point ensures that "good" genes are transmitted from one generation to another while the second property is coherent with the optimization objective of small-sized gene subsets.

Specialized genetic operator of mutation. The mutation operator is used as a source of genetic variation in the population by introducing new genetic information by making local changes in a given chromosome. For binary coded GAs, this is typically realized by flipping the value of some bits $(1 \rightarrow 0$, or $0 \rightarrow 1$ ). In our case, mutation is used for dimension reduction; each application of mutation eliminates a single gene $(1 \rightarrow 0)$. To determine which gene is removed, we use discriminant coefficients obtained from LDA classifier [5]. Given a candidate gene subset, we identify the smallest LDA discriminant coefficient and remove the corresponding gene that is the least informative gene among the current candidate gene subset.

\section{Experiments on Microarray Datasets}

In this section, we use seven public microarray datasets for our experiments (more details in table1). In this table is shown the number of genes, the number of samples and the first publication that has presented an analysis of this dataset.

Our filter-wrapper approach was implemented in octave for Linux O.S. A Fisher LDA classifier is used. In order to test each dataset we conduce 10 independent runs and we retain the best solution found during these 10 executions to have statistically meaningful conclusions. In the first experiment, we focus on the accuracy, so the fitness function is defined with $\alpha=0.50$ (results shown in Figure 2). 
We show in Table 2 the best accuracies (in bold) obtained by other methods and by our filter-wrapper approach on the seven datasets presented previously. An entry with the symbol (-) in this table means that the paper does not treat the corresponding dataset. All the methods reported in this table use a process of cross validation. Each cell contains the classification accuracy and the number of genes when this is available.

Table 1. Description of seven microarray datasets

\begin{tabular}{|c|c|c|c|c|c|}
\hline Dataset & Genes & Samples & Training set & Test set & References \\
\hline Leukemia & 7129 & 72 & 38 & 34 & Golub et al. [6] \\
\hline Colon & 2000 & 62 & 32 & 32 & Alon et al. [2] \\
\hline Lung & 12533 & 181 & 102 & 101 & Gordon et al. [22] \\
\hline Prostate & 12600 & 109 & 54 & 55 & Singh et al. [23 \\
\hline CNS & 7129 & 60 & 30 & 30 & Pomeroy et al. [25] \\
\hline Ovarian & 15154 & 253 & 91 & 162 & Petricoin et al. [26] \\
\hline DLBCL & 4026 & 47 & 23 & 24 & Alizadeh et al. [1] \\
\hline
\end{tabular}

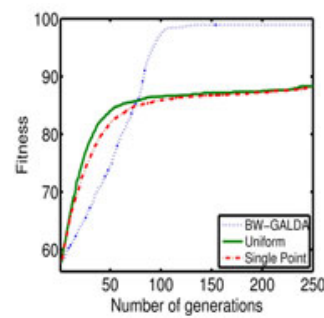

a) Leukemia

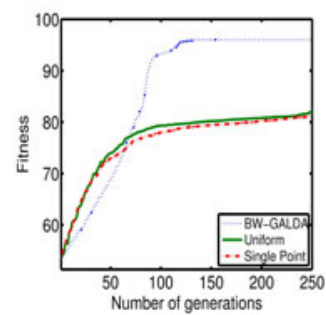

d) CNS

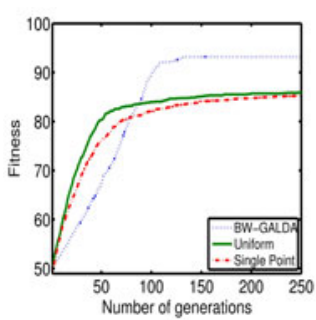

b) Colon

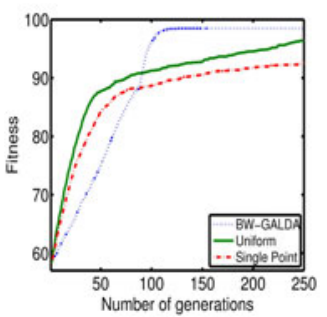

e) Lung

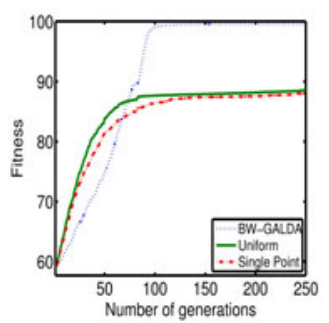

c) DLBCL

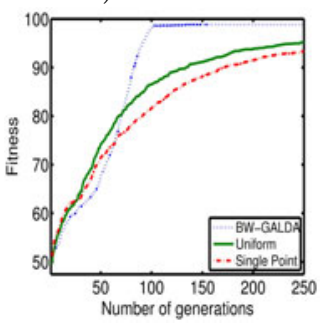

f) Prostate

Fig. 2. Comparison of the best fitness averaged over 10 independent runs with the classical genetic algorithms: uniform crossover and single point crossover. In this experiment was used a $\alpha=0.50$.

According to these observations, it seems clear that our filter-wrapper approach is very competitive in comparison with some top-performing methods. It is difficult to assess this statement by statistical tests since many methods only deal with two datasets. 
Table 2. Comparison of our filter-wrapper approach with the most relevant works on cancer classification

\begin{tabular}{|c|c|c|c|c|c|c|}
\hline Reference & Leukemia & Colon & DLBCL & Lung & Prostate & CNS \\
\hline$[10]$ & 97.5 & 85.0 & - & - & 92.5 & - \\
\hline$[27$ & $100(30)$ & $91.9(30)$ & $98(30)$ & $100(30)$ & $97(30)$ & - \\
\hline$[28$ & 91.1 & 95.1 & - & 93.2 & 73.5 & 88.3 \\
\hline$[29$ & 100 & 93.5 & - & 97.2 & - & - \\
\hline$[30$ & $95.9(25)$ & $87.7(25)$ & $93(25)$ & - & - & - \\
\hline$[31$ & 73.2 & 84.8 & - & - & 86.8 & - \\
\hline$[33$ & $98.6(5)$ & $87(4)$ & - & $100(3)$ & - & - \\
\hline$[34$ & $95.8(20)$ & $100(20)$ & $95.6(20)$ & - & - & - \\
\hline$[35$ & $94.1(35)$ & $83.8(23)$ & - & $91.2(34)$ & - & $65(46)$ \\
\hline$[36$ & $97.1(20)$ & $83.5(20)$ & $93.0(20)$ & - & $91.7(20)$ & $68.5(20)$ \\
\hline$[37$ & $100(30)$ & $90.3(30)$ & $92.2(30)$ & $100(30)$ & $95.2(30)$ & $80(30)$ \\
\hline 11 & $83.8(100)$ & $85.4(100)$ & - & - & - & - \\
\hline$[38$ & $100(4)$ & $93.6(15)$ & - & - & - & - \\
\hline BW-GALDA with $\alpha=0.50$ & $99.3( \pm 2.4)$ & $94.1( \pm 3.2)$ & $99.6( \pm 1.0)$ & $98.6( \pm 2.0)$ & $99.3( \pm 1.5)$ & $97.8( \pm 1.0)$ \\
\hline
\end{tabular}

We show in table 3 and table 4 the most frequent genes obtained by our model for the Leukemia and Colon by using the filters TT, BW and WT. In the first column we show the ID number for each dataset, in the second column we show their frequency and finally in third column is shown the references where is reported these gene in the literature by other well-know models.

Table 3. Summary of 10 first-genes with highest frequency for Leukemia dataset

\begin{tabular}{|c|c|c|c|c|c|c|c|c|}
\hline \multicolumn{3}{|c|}{ FILTER-BW } & \multicolumn{3}{|c|}{ FILTER-TT } & \multicolumn{3}{|c|}{ FILTER-WT } \\
\hline$\overline{\text { Gene-id }}$ & Frequency & References & Gene-id & Frequency & References & Gene-id & Frequency & $\overline{\text { References }}$ \\
\hline 1834 & 915 & & 3847 & 823 & & 3847 & 933 & \\
\hline 4847 & 535 & \begin{tabular}{|l|l|l|l|}
6 & 9 & 8 & 32 \\
\end{tabular} & 1882 & 582 & \begin{tabular}{|l|l|l|}
38 & 9 & 32 \\
\end{tabular} & 1779 & 709 & 9 \\
\hline 1239 & 466 & & 3252 & 507 & 9 & 1882 & 693 & \begin{tabular}{|l|l|l|}
38 & 9 & 32 \\
\end{tabular} \\
\hline 312 & 383 & 8 & 4847 & 368 & \begin{tabular}{|l|l|l|l|}
6 & 8 & 9 & 32 \\
\end{tabular} & 4847 & 584 & \begin{tabular}{|l|l|l|l|}
6 & 8 & 9 & 32 \\
\end{tabular} \\
\hline 1829 & 290 & & 5122 & 367 & & 5122 & 497 & \\
\hline 2242 & 279 & & 5039 & 312 & & 4377 & 386 & 9 \\
\hline 5501 & 279 & & 1834 & 281 & 9 & 1829 & 318 & 9 \\
\hline 4373 & 278 & & 6041 & 277 & 9 & 4373 & 314 & \\
\hline 2288 & 274 & 89 & 1779 & 223 & 9 & 1807 & 295 & \\
\hline 6041 & 230 & 9 & 6169 & 182 & & 2020 & 279 & 9 \\
\hline
\end{tabular}

Table 4. Summary of 10 first-genes with highest frequency for Colon dataset

\begin{tabular}{|c|c|c|c|c|c|c|c|c|}
\hline \multicolumn{3}{|c|}{ FILTER-BW } & \multicolumn{3}{|c|}{ FILTER-TT } & \multicolumn{3}{|c|}{ FILTER-WT } \\
\hline Gene-id & Frequency & References & Gene-id & Frequency & References & Gene-id & Frequency & References \\
\hline 739 & 694 & & 1836 & 532 & & 493 & 879 & 8 \\
\hline 164 & 670 & & 548 & 526 & & 1836 & 737 & \\
\hline 576 & 513 & & 792 & 407 & 8 & 1873 & 284 & \\
\hline 625 & 464 & 38 & 377 & 392 & & 765 & 277 & \\
\hline 399 & 391 & & 493 & 381 & 8 & 377 & 276 & \\
\hline 1472 & 384 & & 18 & 362 & 8 & 897 & 252 & 38 \\
\hline 26 & 297 & & 67 & 330 & & 792 & 227 & 8 \\
\hline 619 & 296 & & 765 & 301 & & 180 & 216 & \\
\hline 451 & 294 & & 517 & 288 & & 267 & 205 & \\
\hline 249 & 280 & 38 & 823 & 287 & & 689 & 203 & \\
\hline
\end{tabular}

In table 5 are shown the number of genes selected by our model in the seven microarray datasets. In this way we could obtain a smaller number of genes with 
a perfect classification accuracy in 5 of seven datasets. Those minimum set of genes can be used to differentiate the two types of diseases(p.e. ALL/AML for the Leukemia dataset). For the others datasets we obtain a very good classification for the Colon, CNS and Lung cancer.

Table 5. Best classification rates for seven public dataset using small gene subset with the filters: BW, TT and WT

\begin{tabular}{|l|c|c|c|}
\hline Data sets & $\begin{array}{c}\text { filter-BW } \\
\text { Accuracy(Number of genes) }\end{array}$ & $\begin{array}{c}\text { filter-TT } \\
\text { Accuracy(Number of genes) }\end{array}$ & $\begin{array}{c}\text { filter-WT } \\
\text { Accuracy(Number of genes) }\end{array}$ \\
\hline Leukemia & $99.3( \pm 2.3)$ & $99.3( \pm 3)$ & $99.3( \pm 2.6)$ \\
Colon & $94.1( \pm 3)$ & $93.1( \pm 3.1)$ & $94.1( \pm 2.6)$ \\
DLBCL & $99.6( \pm 1)$ & $99.3( \pm 3.1)$ & $99.3( \pm 1)$ \\
CNS & $97( \pm 1)$ & $97.3( \pm 1)$ & $98.6( \pm 1)$ \\
Lung & $98.6( \pm 2)$ & $98.6( \pm 2)$ & $98.6( \pm 2.1)$ \\
Prostate & $99.3( \pm 2)$ & $99.3( \pm 1.7)$ & $99.3( \pm 1.9)$ \\
Ovarian & $97.8( \pm 1)$ & $99.3( \pm 2.2)$ & $96.3( \pm 1.0)$ \\
\hline
\end{tabular}

\section{Conclusions}

In this paper we propose a new combined filter-wrapper with specialized genetic operators for the gene selection and classification of microarray gene expression. The propose approach begins with a filter that pre-selects a first set of genes (about $p=150$ in this paper). To further explore the combinations of these genes, we rely on a hybrid Genetic Algorithm combined with Fishers Linear Discriminant Analysis. In this LDA-GA, LDA is used not only to assess the fitness of a candidate gene subset, but also to inform the crossover and mutation operators. This GA and LDA hybridization makes the genetic search highly efficient for identifying small and informative gene subsets.

We use a double function fitness that provides an interesting way for the LDAGA to explore the gene subset space either for the minimization of the selected genes or for the maximization of the prediction accuracy.

We have extensively evaluated our filter-wrapper approach on seven public datasets using a rigorous 10-fold cross-validation process. A large comparison was carried out with 13 state-of-art algorithms that are based on a variety of methods. The results clearly show the competitiveness of our filter-wrapper approach. For all the datasets, our approach is able to select small gene subsets while ensuring the best or the second best classification rate. The proposed approach has another practically useful feature for biological analysis. In fact, instead of producing a single solution (gene subset), our approach can easily and naturally provide multiple non-dominated solutions that constitute valuable candidates for further biological investigations.

\section{Acknowledgement}

This work is supported by the PROMEP project ITAPI-EXB-000. We would like to thank the referees for their helpful questions, comments and suggestions. 


\section{References}

1. Alizadeh, A., Eisen, M.B., et al.: Distinct types of diffuse large (b)-cell lymphoma identified by gene expression profiling. Nature 403, 503-511 (2000)

2. Alon, U., Barkai, N., et al.: Broad patterns of gene expression revealed by clustering analysis of tumor and normal colon tissues probed by oligonucleotide arrays. PNAS 96, 6745-6750 (1999)

3. Ben-Dor, A., Bruhn, L., et al.: Tissue classification with gene expression profiles. Journal of Computational Biology 7(3-4), 559-583 (2000)

4. Bonilla-Huerta, E., Duval, B., Hao, J.-K., et al.: A hybrid GA/SVM approach for gene selection and classification of microarray data. In: Rothlauf, F., Branke, J., Cagnoni, S., Costa, E., Cotta, C., Drechsler, R., Lutton, E., Machado, P., Moore, J.H., Romero, J., Smith, G.D., Squillero, G., Takagi, H. (eds.) EvoWorkshops 2006. LNCS, vol. 3907, pp. 34-44. Springer, Heidelberg (2006)

5. Bonilla-Huerta, E., Duval, B., Hao, J.-K., et al.: Gene selection for microarray by a LDA-based genetic algorithms. In: Chetty, M., Ngom, A., Ahmad, S. (eds.) PRIB 2008. LNCS (LNBI), vol. 5265, pp. 250-261. Springer, Heidelberg (2008)

6. Golub, T., Slonim, D., et al.: Molecular classification of cancer: Class discovery and class prediction by gene expression monitoring. Science 286, 531-537 (1999)

7. Dudoit, S., Fridlyand, J., Speed, T.: Comparison of discrimination methods for the classification of tumors using gene expression data. JASA 97, 77-87 (2002)

8. Cai, R., Hao, Z., Yang, X., Wen, W.: An efficient gene selection algorithm based on mutual information. Neurocomputing 26(3), 243-250 (2008)

9. Liao, C., Li, S., Luo, Z.: Gene selection for cancer classification using Wilcoxon Rank Sum Test and Support Vector Machine. In: International Conference on Computation Intelligence and Security, pp. 368-373 (2006)

10. Ye, J., Li, T., et al.: Using uncorrelated discriminant analysis for tissue classification with gene expression data. IEEE/ACM Trans. Comput. Biology Bioinform. 1(4), 181-190 (2004)

11. Yue, F., Wang, K., Zuo, W.: Informative gene selection and tumor classification by null space lda for Microarray data. In: Chen, B., Paterson, M., Zhang, G. (eds.) ESCAPE 2007. LNCS, vol. 4614, pp. 435-446. Springer, Heidelberg (2007)

12. Guyon, I., Elisseeff, A.: An introduction to variable and feature selection. JMLR 3, 1157-1182 (2003)

13. Furey, T.S., Cristianini, N., et al.: Support vector machine classification and validation of cancer tissue samples using microarray expression data. Bioinformatics 16(10), 906-914 (2000)

14. Li, L., Weinberg, C.R., et al.: Gene selection for sample classification based on gene expression data: study of sensitivity to choice of parameters of the GA/KNN method. Bioinformatics 17(12), 1131-1142 (2001)

15. Jourdan, L.: Metaheuristics for knowledge discovery: Application to genetic data, PhD thesis, University of Lille (2003) (in French)

16. Peng, S., Xu, Q., et al.: Molecular classification of cancer types from microarray data using the combination of genetic algorithms and support vector machines. FEBS Letter 555(2), 358-362 (2003)

17. Reddy, A.R., Deb, K.: Classification of two-class cancer data reliably using evolutionary algorithms, Technical Report. KanGAL (2003)

18. Guyon, I., Weston, J., et al.: Gene selection for cancer classification using support vector machines. Machine Learning 46(1-3), 389-422 (2002) 
19. Saeys, Y., Aeyels, S., et al.: Feature selection for splice site prediction: A new method using eda-based feature ranking. BMC Bioinformatics, 5-64 (2004)

20. Goh, L., Song, Q., Kasabov, N.: A novel feature selection method to improve classification of gene expression data. In: Proc. of the 2nd Asia-Pacific Conference on Bioinformatics, ACS, Darlinghurst, Australia, pp. 161-166 (2004)

21. Hall, M., Holmes, G.: Benchmarking attribute selection techniques for discrete class data mining. IEEE Trans. Knowl. Data Eng. 15(6), 1437-1447 (2003)

22. Gordon, G.J., Jensen, R.V., et al.: Translation of microarray data into clinically relevant cancer diagnostic tests using gene expression ratios in lung cancer and mesothelioma. Cancer Research 17(62), 4963-4967 (2002)

23. Singh, D., Febbo, P., et al.: Gene expression correlates of clinical prostate cancer behavior. Cancer Cell 1, 203-209 (2002)

24. Piqué-Regí, R., Ortega, A., Asgharzadeh, S.: Sequential diagonal linear discriminant analysis (SeqDLDA) for microarray classification and gene identification. Computational Systems and Bioinformatics (2005)

25. Pomeroy, S.L., Tamayo, P., et al.: Prediction of central nervous system embryonal tumour outcome based on gene expression. Nature 415, 436-442 (2002)

26. Petricoin, E.F., Ardekani, A.M., et al.: Use of proteomic patterns in serum to identify ovarian cancer. Lancet 359, 572-577 (2002)

27. Liu, H., Li, J., Wong, L.: A comparative study on feature selection and classification methods using gene expression profiles and proteomic pattern. Genomic Informatics 13, 51-60 (2002)

28. Tan, F., Fu, X., et al.: Improving Feature Subset Selection Using a Genetic Algorithm for Microarray Gene Expression Data. In: CEC-IEEE, pp. 2529-2534 (2006)

29. Ding, C., Peng, H.: Minimum redundancy feature selection from Microarray gene expression data. Bioinformatics and Computational. Biology 3(2), 185-206 (2005)

30. Cho, S.B., Won, H.H.: Cancer classification using ensemble of neural networks with multiple significant gene subsets. Applied Intelligence 26(3), 243-250 (2007)

31. Yang, W.H., Dai, D.Q., Yan, H.: Generalized discriminant analysis for tumor classification with gene expression data. Machine Learning and Cybernetics 1, 4322-4327 (2006)

32. Yang, P., et al.: A multi-filter enhanced genetic ensemble system for gene selection and sample classification of microarray data. BMC Bioinformatics 11(suppl. 1), S6 (2010)

33. Peng, Y., Li, W., Liu, Y.: A hybrid approach for biomarker discovery from Microarray gene expression data. Cancer Informatics 2, 301-311 (2006)

34. Wang, Z., Palade, V., Xu, Y.: Neuro-fuzzy ensemble approach for Microarray cancer gene expression data analysis. In: Proc. E. Fuzzy Systems, pp. 241-246 (2006)

35. Pang, S., Havukkala, I., et al.: Classification consistency analysis for bootstrapping gene selection. Neural Computing and Applications 16, 527-539 (2007)

36. Li, G.Z., Zeng, X.Q., et al.: Partial least squares based dimension reduction with gene selection for tumor classification. In: BIBE-IEEE, pp. 1439-1444 (2007)

37. Zhang, L., Li, Z., Chen, H.: An effective gene selection method based on relevance analysis and discernibility matrix. In: Zhou, Z.-H., Li, H., Yang, Q. (eds.) PAKDD 2007. LNCS (LNAI), vol. 4426, pp. 1088-1095. Springer, Heidelberg (2007)

38. Li, S., Wu, X., Hu, X.: Gene selection using genetic algorithm and support vectors machines. Soft Computing 12(7), 693-698 (2008) 